\title{
Elevated expression of Nodal and YAP1 is associated with poor prognosis of gastric adenocarcinoma
}

\author{
Ping $\mathrm{Li}^{1} \cdot \operatorname{Dan} \mathrm{Sun}^{1} \cdot \mathrm{Xiaoting} \mathrm{Li}^{2} \cdot \mathrm{Yingjian} \mathrm{He}^{2} \cdot$ Wenhui $\mathrm{Li}^{1} \cdot \mathrm{Jing} \mathrm{Zhao}^{1} \cdot$ \\ Ying Wang ${ }^{1} \cdot$ Huan Wang ${ }^{1} \cdot$ Yan Xin ${ }^{1}$
}

Received: 13 February 2016 / Accepted: 7 June 2016 / Published online: 20 June 2016

(C) The Author(s) 2016. This article is published with open access at Springerlink.com

\begin{abstract}
Purpose To investigate the correlation between Nodal and YAP1 expression and the clinicopathological characteristics of gastric adenocarcinoma (GAC).

Methods Quantitative real-time RT-PCR, Western blot, and immunohistochemistry were performed to measure Nodal and YAP1 mRNA and protein in 20 fresh frozen samples and 220 paraffin-embedded GAC tissues with their paired non-tumor mucosa (PNTM). The prognostic values of Nodal and YAP1 were evaluated in 161 GAC patients using univariate and multivariate analyses.

Results Both mRNA and protein expression of Nodal and YAP1 were significantly increased in GAC compared to PNTM $(P<0.05)$. Immunohistochemistry showed that Nodal was more highly expressed in $56.4 \%$ GAC samples compared to PNTM; additionally, Nodal expression correlated with depth of tumor invasion, lymph node metastasis, and distant metastasis (all $P<0.05$ ). There was no association between Nodal and YAP1 in GAC $(P=0.171)$. Kaplan-Meier analysis showed that the outcome of Nodalhigh patients was significantly worse than those with low
\end{abstract}

Dr. Dan Sun and Dr. Ping Li are listed as the parallel first authors.

Yan Xin

yxin@mail.cmu.edu.cn

1 Laboratory of Gastrointestinal Onco-Pathology, Cancer Institute and General Surgery Institute, The First Affiliated Hospital of China Medical University, 155 Nanjing North Street, Heping District, Shenyang 110001, Liaoning Province, China

2 Key Laboratory of Carcinogenesis and Translational Research (Ministry of Education), Peking University Cancer Hospital \& Institute, No. 52 Fucheng Road, Haidian District, Beijing 100142, China
Nodal expression $\left(\chi^{2}=30.452, P<0.001\right)$. Cox multivariate regression showed that high Nodal expression was an independent risk factor affecting the prognosis of GAC patients $(P=0.000, \mathrm{RR}=2.976)$. Furthermore, patients with tumors in which both Nodal and YAP1 were expressed at high levels had the worst prognosis.

Conclusions Elevated Nodal expression is a marker of poor prognosis in gastric cancer. Patient outcome is further worsened if Nodal and YAP1 are both expressed in the same tumor. The datas we present here suggest that the inhibition of Nodal signaling may represent a new therapeutic strategy for the treatment of gastric adenocarcinoma.

Keywords Nodal · YAP1 - Gastric adenocarcinoma · Prognosis

\section{Introduction}

Nodal, a member of the transforming growth factor- $\beta$ (TGF- $\beta$ ) superfamily, regulates many diverse cellular processes, including the growth and differentiation of embryonic stem cells and the plasticity and invasiveness of malignant tumor cells. As such, Nodal helps regulate the pluripotency of embryonic stem cells and cancer cells (James et al. 2005). Nodal signaling also plays a critical role during the development of vertebrates, echinoderms, and protostomes. During early vertebrate development, Nodal is necessary and sufficient for endomesoderm induction and patterning of anteroposterior (AP), dorsoventral (DV), and left-right (LR) axes. During echinoderm development, Nodal is important for determining DV and LR asymmetry. In contrast, in protostomes, Nodal signaling is only involved in establishing LR asymmetry (Röttinger et al. 2015). While Nodal is an essential molecule early in 
development, it is thought to be largely absent from adult tissues; the few exceptions include some adult stem cell populations and some highly dynamic reproductive tissues (Aykul et al. 2015). Nodal signaling is regulated, at least in part, by secreted proteins Lefty and Cerberus. During development, Nodal exerts its functions both by regulating its own expression and via unilateral activation of downstream genes, such as Lefty and Pitx. In turn, Lefty and Pitx regulate expression of several additional genes, many of which have roles in proliferation and differentiation (Soukup et al. 2015). For example, one study showed that exposure of tumor cells to an hESC microenvironment containing Lefty resulted in decreased Nodal expression, colony forming ability, and tumorigenicity (Postovit et al. 2008). Additionally, Cerberus, another Nodal inhibitor that functions by preventing the interaction between Nodal and its receptor, profoundly suppresses migration, invasion, and colony formation ability of breast cancer cells (Aykul et al. 2015). Studies such as these suggest that approaches to decrease Nodal expression may have some therapeutic value. This is particularly relevant since several earlier studies have established that Nodal is up-regulated in various cancers, including breast cancer (Kirsammer et al. 2004), melanoma (Strizzi et al. 2015), endometrial cancer (Cruz et al. 2015), and pancreatic cancer (Kong et al. 2015). Nodal appears to play many roles during oncogenesis as well; indeed, Nodal has been associated with increased tumor angiogenesis, invasion, and metastasis.

YAP1 is a transcriptional coactivator of the Hippo pathway with roles in tumorigenesis. Since it regulates tumor cell proliferation and apoptosis, YAP1 has been described as a candidate oncogene (Sudol 1994). Several studies have reported increased expression of Nodal and YAP1 in many tumor types, including melanoma, liver cancer, breast cancer, ovarian cancer, and glioma. This increased expression in cancer suggests that Nodal and YAP1 might play important roles in tumor proliferation, angiogenesis, invasion, and metastasis.

Importantly, previous studies have reported cross talk between components of the Hippo and TGF- $\beta$ pathways, both at the level of intracellular signaling and transcriptional regulation (Grannas et al. 2015). As one example in endothelial cells, TGF- $\beta$ induces nuclear localization of a Smad2/3/4 complex, which activates expression of Snail, Twist1, and Slug, key transcription factors required for epithelial-mesenchymal transition (EMT). YAP1 interacts with this complex, and loss of YAP1 disrupts the TGF- $\beta$ mediated up-regulation of Snail, Twist1, and Slug (Zhang et al. 2014). These findings highlight the potential importance of cross talk between these two pathways for cellular processes. Despite this, to date, potential roles for Nodal and YAP1 have not been reported in gastric adenocarcinoma (GAC). In this study, we measured mRNA and protein expression of both Nodal and YAP1 in GAC and paired non-tumor mucosa (PNTM). We examined possible correlation between expression of these factors and several clinicopathological variables. The data we present here suggest that inhibition of Nodal signaling may represent a new therapeutic strategy for the treatment of gastric adenocarcinoma.

\section{Materials and methods}

\section{Samples and clinicopathological data}

Two hundred and twenty surgically resected gastric adenocarcinoma (GAC) specimens and paired non-tumor mucosa (PNTM) (obtained at a location greater than $5 \mathrm{~cm}$ away from the edge of the primary tumor) were collected at the First Affiliated Hospital of China Medical University. Specimens were fixed in $10 \%$ neutral formalin. Conventional wax baits were cut consecutively into $4 \mu \mathrm{m}$ sections and then subject to conventional H\&E staining to determine pathological diagnosis. Twenty additional fresh frozen specimens from postoperative GAC and corresponding PNTM were collected and preserved at $-80{ }^{\circ} \mathrm{C}$ for qRTPCR and Western blot. None of the patients had received radiotherapy or chemotherapy prior to surgery. None of the patients received adjuvant or palliative therapy either. Clinical and pathological data for all patient samples used in the study are listed in Table 2. Informed consent was obtained from the Ethics Committee in the First Affiliated Hospital of China Medical University.

\section{RNA extraction and quantitative real-time reverse transcriptase PCR (qRT-PCR)}

Total RNA was isolated from 20 tumor/non-tumor paired tissue samples using the EASYspin Plus kit (Aidlab Biotechnologies, Beijing) according to the manufacturer's instructions. RNA quantity and quality were analyzed with Nanodrop 1000 (Thermo Fisher Scientific). Isolated RNA was reversetranscribed to cDNA using the PrimeScript ${ }^{\circledR}$ RT reagent kit (TaKaRa, Dalian, China) according to the manufacturer's protocol. Primers to measure the expression of genes of interest were designed using Prime5 software. Primer sequences are as follows: Nodal-Sense 5'-ACATCATCCGCAGCCTACA-3', anti-sense $5^{\prime}$-AGCCCATGCCAGATCCTC-3', YAP1Sense $5^{\prime}$-TACGATACAAGGCTGTTAGAGAG- $3^{\prime}$, antisense 5'-TTGAGATGCATGCTTTGCATAC-3'; GAPDHsense $5^{\prime}$-GAAGGTGAAGGTCGGAGTC-3', and anti-sense 5'-GAAGATGGTGATGGGATTTC- ${ }^{\prime}$.

Each PCR was prepared in a total volume of $10 \mu \mathrm{l}$, which included each primer, diluted cDNA templates, and SYBR $^{\circledR}$ Premix TaqTM II (TaKaRa, Dalian, China). 
Cycling conditions for qRT-PCR were as follows: $95^{\circ} \mathrm{C}$ for $30 \mathrm{~s}$ followed by 40 cycles of $95^{\circ} \mathrm{C}$ for $5 \mathrm{~s}$ and annealing at $58.5^{\circ} \mathrm{C}$ for $30 \mathrm{~s}$. Melting curves were generated by $95{ }^{\circ} \mathrm{C}$ for $15 \mathrm{~s}, 60{ }^{\circ} \mathrm{C}$ for $30 \mathrm{~s}$, and $95{ }^{\circ} \mathrm{C}$ for $15 \mathrm{~s}$. Data were calculated using the $2-\Delta \Delta \mathrm{Ct}$ method. The mRNA level of each sample was normalized to that of GAPDH mRNA, and each cDNA sample was run in triplicate.

\section{Protein extraction and Western blot}

Protein was harvested from samples using lysis buffer containing phenylmethylsulfonylfluoride (PMSF) at $4{ }^{\circ} \mathrm{C}$. Total extracted proteins were quantified using a BCA protein assay kit (ComWin Biotech, Beijing, China). Samples were boiled at $95{ }^{\circ} \mathrm{C}$ for $5 \mathrm{~min}$, and then, $50 \mu \mathrm{g}$ of protein was electrophoretically separated using $10 \%$ SDS-PAGE gels (Invitrogen). Following electrophoresis, samples were transferred to a polyvinylidene difluoride (PVDF) membrane via wet transfer. PVDF membranes were blocked with $5 \%$ nonfat dry milk in Tris-phosphate buffer containing $0.05 \%$ Tween 20 (TBS-T) for $1 \mathrm{~h}$ at room temperature, and then incubated with primary antibodies at room temperature overnight. The following primary antibodies were used in this study: rabbit anti-Nodal polyclonal antibody (working dilution 1:1000, MILLIPORE), rabbit anti-YAP1 monoclonal antibody (1:1000, Abcam, Britain), and $\beta$-actin (1:1000, ZhongShan-Golden Bridge, Beijing), which was used as an internal loading control. Membranes were then incubated with horseradish peroxidaseconjugated anti-mouse or anti-rabbit secondary antibodies (1:1000, ZhongShan-Golden Bridge, Beijing) and visualized using an enhanced chemiluminescence (ECL) detection system. Blots were visualized using an ECL Kit (ComWin Biotech, Beijing, China) and quantified using ImageJ software. Results are expressed as fold change compared to the control values. All experiments were performed at least three times.

\section{Tissue microarray and immunohistochemistry}

Two hundred and twenty GAC and paired normal mucosa samples were fixed in $10 \%$ formalin, embedded in paraffin, and cut into $4 \mu \mathrm{m}$-thick sections. All samples were evaluated by two experienced pathologists, who confirmed the diagnoses and marked various target lesions. Six blocks of tissue microarray containing 220 gastric adenocarcinomas and their corresponding normal gastric mucosa were constructed using microarrayer (USA). The six blocks of tissue microarray were then cut to yield $4-\mu \mathrm{m}$ serial sections and placed on glass slides using an adhesive-tape transfer system for immunohistochemistry.

Nodal and YAP1 protein expression were measured using two-step immunohistochemistry. Rabbit anti-Nodal polyclonal antibody (working dilution 1:50) and rabbit anti-YAP1 monoclonal antibody (working dilution 1:50) were purchased from MILLIPORE and Abcam, respectively. The DAB kit was purchased from Fuzhou Maixin Company (Fuzhou, China). The PV-9000 kit was obtained from Beijing Zhongshan Goldenbridge Biotechnology Company (Beijing, China). Tissue microarray slides were deparaffinized in xylene and hydrated with alcohol before being placed in $3 \% \mathrm{H}_{2} \mathrm{O}_{2}$ methanol blocking solution. This was then followed by heat-induced antigen retrieval. The slides were incubated with primary antibodies overnight at $4{ }^{\circ} \mathrm{C}$, stained with the PV-9000 detection system, and counterstained with hematoxylin. All procedures were carried out according to the manufacturer's instructions. For negative controls, sections were treated with $0.01 \mathrm{M}$ phosphatebuffered saline instead of primary antibodies.

\section{Assessment of immunohistochemistry staining}

Nodal or YAP1 positivity was defined as samples displaying clear brown granules either in the cytoplasm or nucleus. The expression of Nodal and YAP1 was determined by assigning proportion and intensity scores to both tumor cells and adjacent gastric mucosa epithelial cells. This type of staining analysis has been previously described (Vandeputte et al. 2002). The proportion score was assigned based on the proportion of positive cells $(0$, none; $1, \leq 10 \% ; 2,11-25 \% ; 3$, $26-50 \% ; 4,>50 \%)$. The intensity score was assigned based on comparison to YAP1-positive or Nodal-positive internal controls ( 0 , none; 1 , weak; 2 , intermediate; 3 , strong). The total score for Nodal or YAP1 expression, ranging from 0 to 12 , was the product of the proportion and intensity scores. The expression was categorized as negative (0), (-); low (score 1-3), (1+); intermediate (score 4-6), (2+); or high (score 7-12), (3+). According to the above-mentioned criteria, samples scoring $0-1+$ were considered the low-expression group, and samples scoring $2+$ to $3+$ were considered the high-expression group for subsequent statistical analysis.

\section{Statistical analysis}

Categorical data have been described using frequencies and percentages. Continuous data have been presented as means with standard deviations for normally distributed data. Statistical analysis was performed using SPSS 17.0 software. Either Chi-square test or Fisher's exact test was used to differentiate the rates of different groups. Time-toevent data were estimated by the Kaplan-Meier method and analyzed with the log-rank test. The cumulative overall survival rates were calculated using life table techniques, illustrated by Kaplan-Meier plots. The multivariable analysis model was fit using a Cox proportional hazards regression model using SPSS 17.0. The enter method was used to determine a final Cox model. All statistical analyses were two-sided, and significance was assigned at $\alpha=0.05$. 

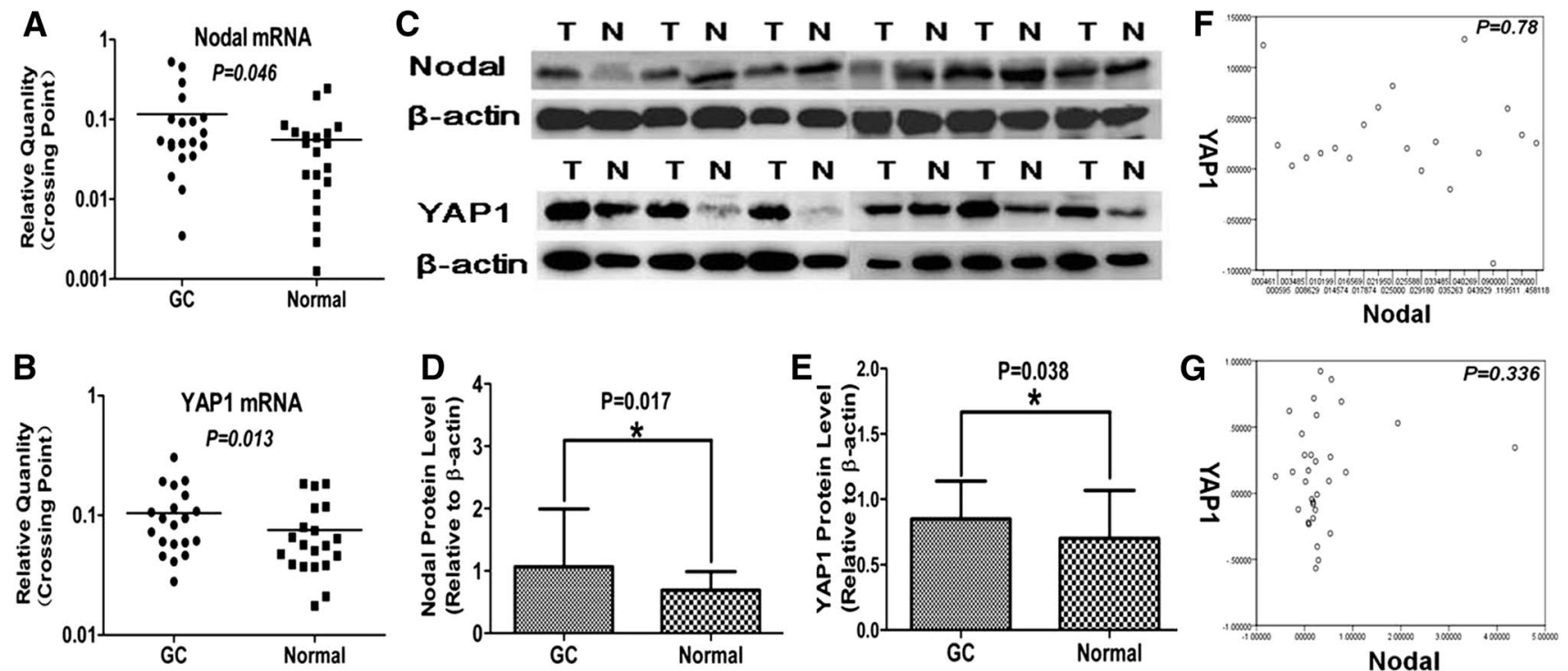

Fig. 1 Expression of Nodal and YAP1 mRNA and protein in GAC. Nodal mRNA (a) and YAP1 mRNA (b) were significantly up-regulated in GAC compared with PNTM $(P<0.05)$; similar results were

found when examining protein expression $(\mathbf{c}-\mathbf{e})$. However, there was no correlation between Nodal and YAP1 expression at either the mRNA (f) or protein (g) level

\section{Results}

\section{Nodal and YAP1 mRNA expression are significantly up-regulated in GAC}

To determine expression levels of Nodal and YAP1, we performed qRT-PCR using RNA isolated from GAC samples and from adjacent normal gastric mucosa. Nodal mRNA was significantly higher in GAC tissue compared to paired normal mucosa $(P=0.046)$. Similarly, YAP1 mRNA expression was also elevated in GAC specimens compared to paired normal mucosa $(P=0.013)$ (Fig. 1; Table 1).

\section{Nodal and YAP1 protein expression are significantly up-regulated in GAC}

We next performed Western blot from GAC samples and their matched normal tissue to determine protein expression of Nodal and YAP1. Consistent with our mRNA analysis, we found significantly increased protein expression of both Nodal $(P=0.017)$ and YAP1 $(P=0.038)$ in GAC tissues compared to matched normal mucosa (Fig. 1; Table 1).

\section{Expression of Nodal and YAP1 are not correlated in GAC}

Since both Nodal and YAP1 are elevated in GAC samples compared to normal mucosa, we next determined if there was any correlation between expressions of these two factors. We found no correlation between Nodal and YAP1 at

Table 1 Nodal and YAP1 mRNA and protein expression in GC and paired normal mucosa

\begin{tabular}{llrlrl}
\hline Groups & $n$ & Nodal expression & $P$ & YAP1 expression & $P$ \\
\hline mRNA & & & $\mathbf{0 . 0 4 6}$ & & $\mathbf{0 . 0 1 3}$ \\
GC & 20 & $0.126 \pm 0.177$ & & $0.105 \pm 0.069$ & \\
Normal & 20 & $0.056 \pm 0.063$ & & $0.075 \pm 0.052$ & \\
Protein & & & $\mathbf{0 . 0 1 7}$ & & $\mathbf{0 . 0 3 8}$ \\
GC & 32 & $1.0699 \pm 0.9241$ & & $0.8494 \pm 0.2893$ & \\
Normal & 32 & $0.6929 \pm 0.2965$ & & $0.7012 \pm 0.3667$ & \\
\hline
\end{tabular}

Bold values indicates that statistical significance of $P$ value is less than 0.05

either the mRNA $(P=0.78)$ or protein $(P=0.336)$ level in either GAC or paired normal tissue expression (Fig. 1).

\section{High Nodal protein expression is associated with poor clinicopathological variables in GAC}

We next wanted to determine if there was any correlation between Nodal expression and clinicopathological variables in GAC. Immunohistochemistry showed that Nodal protein was expressed both in the cytoplasm and nucleus of GAC samples. A total of $56.4 \%$ (124/220) of GAC samples stained positive for Nodal, in contrast to the only $4.3 \%(8 / 186)$ of paired normal tissue showing a positive stain $(P<0.01)$. Furthermore, Nodal expression in GAC correlated with depth of tumor invasion, lymph node metastasis, distant metastasis, and TNM staging (all $P<0.005$ ) (Fig. 2; Table 2). 

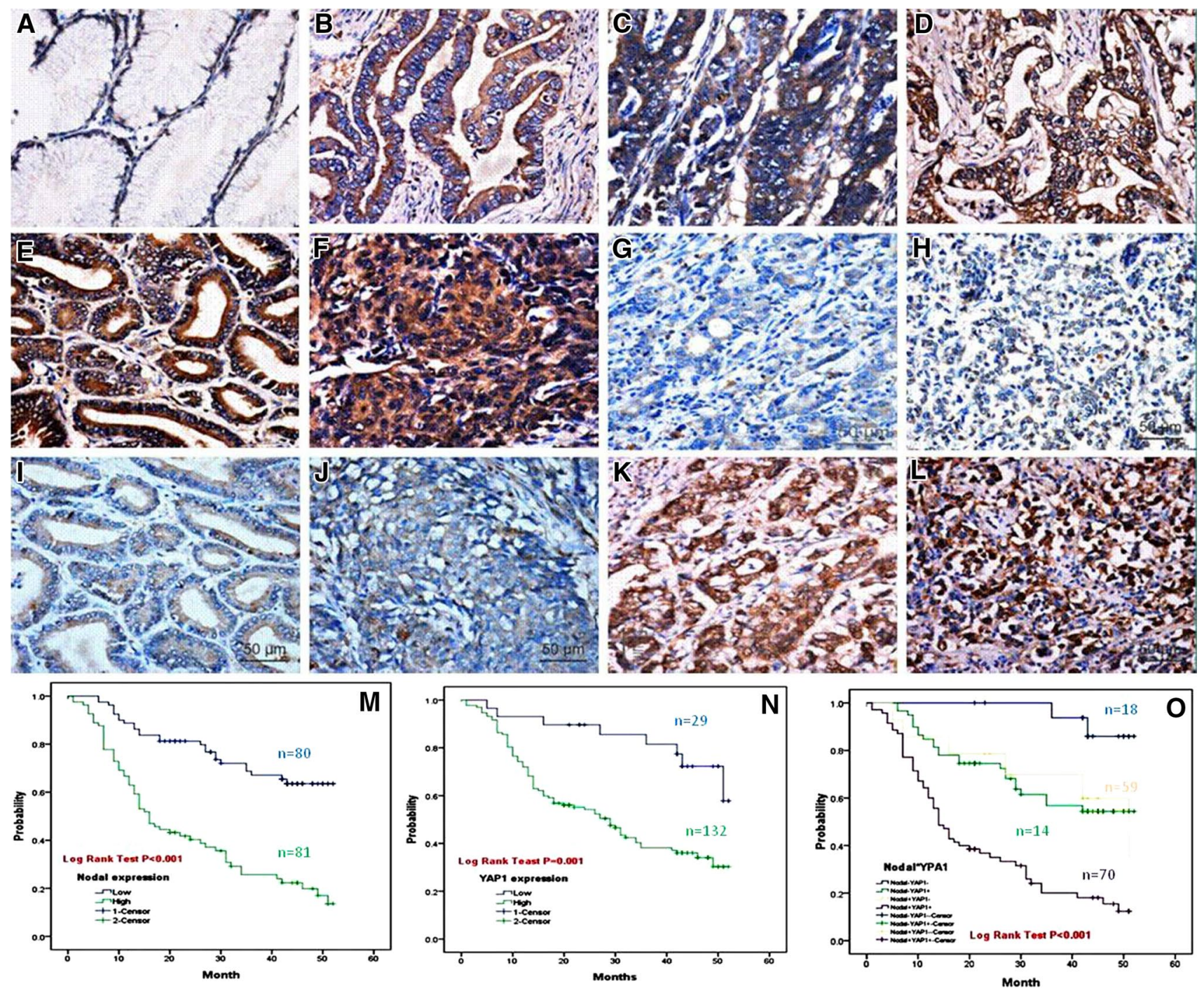

Fig. 2 Correlation between expression of Nodal and YAP1 protein and prognosis of patients with GAC. Compared with the PNTM (a), Nodal expression was significantly up-regulated in GAC $(\mathbf{b}-\mathbf{f})$. Nodal expression (e-h) was not correlated with YAP1 expression (i-l) in the same case of GAC (e vs. i, f vs. $\mathbf{j}, \mathbf{g}$ vs. $\mathbf{k}, \mathbf{h}$ vs. l). Two-step IHC $\times 400$. Kaplan-Meier analyses of the effects of Nodal and YAP1 expressions on patient survival in GAC showed that overall survival for patients with high Nodal expression was significantly worse than that with low expression $(P=0.000, \mathbf{m})$. Overall survival for patients with YAP1 high-expression was significantly worse than that in the YAP1 low-expression group $(P=0.001, \mathbf{n})$. Furthermore, there was an interaction between Nodal and YAP1 expression on GAC outcome, as patients with tumors with high expression of both Nodal and YAP1 had the worst overall prognosis (o)

\section{High Nodal expression is associated with poor prognosis in GAC}

In this study, a total of 161 cases were followed up for 52 months after surgery. The median survival time was $34 \pm 4.79$ months. A total of 88 deaths occurred during follow-up, and all causes of death were cancer-related. Kaplan-Meier survival curves were plotted, and a logrank test was used to evaluate the effect of high Nodal expression on patient outcome. The mean survival time of patients in the Nodal-high group was $23.58 \pm 1.98$ months, significantly shorter than in the Nodal-low group
(40.90 \pm 1.88 months $)(P<0.001)$. These results indicate that high Nodal expression is associated with poor prognosis in gastric adenocarcinoma (Fig. 2m; Table 3).

\section{Patients with GAC tumors expressing both Nodal and YAP1 exhibit the worst overall prognosis}

To evaluate potential interaction between Nodal and YAP1 on GAC prognosis, patients were divided into four groups according to their Nodal and YAP1 expression status. The groups consisted of patients with tumors that were $\mathrm{Nodal}^{-} \mathrm{YAP}^{-}$, $\mathrm{Nodal}^{-} \mathrm{YAP}^{+}, \mathrm{Nodal}^{+} \mathrm{YAP}^{-}$, and $\mathrm{Nodal}^{+} \mathrm{YAP}^{+}$. 
Table 2 Correlation between Nodal and YAP1 expression and clinicopathological features in GC

\begin{tabular}{|c|c|c|c|c|c|c|}
\hline \multirow[t]{2}{*}{ Variable } & \multirow[t]{2}{*}{$n$} & \multicolumn{2}{|c|}{ Nodal expression } & \multirow[t]{2}{*}{ HPR $(\%)$} & \multirow[t]{2}{*}{$\chi^{2}$} & \multirow[t]{2}{*}{$P$ value } \\
\hline & & Low & High & & & \\
\hline Age (year) & & & & & 3.696 & 0.055 \\
\hline$\leq 55$ & 82 & 43 & 39 & 47.6 & & \\
\hline$>55$ & 138 & 54 & 84 & 60.9 & & \\
\hline Gender & & & & & 0.018 & 0.892 \\
\hline Female & 67 & 30 & 37 & 55.2 & & \\
\hline Male & 153 & 67 & 85 & 56.2 & & \\
\hline Borrmann's types & & & & & 0.26 & 0.61 \\
\hline $\mathrm{I}+\mathrm{II}$ & 30 & 12 & 18 & 60.0 & & \\
\hline $\mathrm{III}+\mathrm{IV}$ & 189 & 85 & 104 & 55.0 & & \\
\hline WHO's histology types & & & & & 8.930 & 0.159 \\
\hline Papillary. Ade. & 8 & 3 & 5 & 62.5 & & \\
\hline \multicolumn{7}{|l|}{ Tubular Ade. } \\
\hline Well Diff. & 14 & 9 & 5 & 35.7 & & \\
\hline Moderately Diff. & 74 & 36 & 38 & 51.4 & & \\
\hline Poorly Diff. & 99 & 35 & 64 & 64.6 & & \\
\hline Un-differentiated car. & 3 & 1 & 2 & 66.7 & & \\
\hline Signet ring cell Car. & 7 & 5 & 2 & 28.6 & & \\
\hline Mucinous Ade. & 15 & 8 & 7 & 46.7 & & \\
\hline Lauren's types & & & & & 0.735 & 0.692 \\
\hline Intestinal & 80 & 38 & 42 & 53.5 & & \\
\hline Diffuse & 102 & 42 & 60 & 58.8 & & \\
\hline Mixed & 38 & 17 & 21 & 55.3 & & \\
\hline Depth of invasion & & & & & 8.899 & 0.003 \\
\hline $\mathrm{T} 1+\mathrm{T} 2$ & 36 & 24 & 12 & 33.3 & & \\
\hline $\mathrm{T} 3+\mathrm{T} 4$ & 184 & 73 & 111 & 60.3 & & \\
\hline Ln metastasis & & & & & 19.669 & $<0.001$ \\
\hline No & 60 & 41 & 19 & 31.7 & & \\
\hline $\mathrm{N} 1-3$ & 160 & 56 & 104 & 65.0 & & \\
\hline Distant metastasis & & & & & 23.815 & $<0.001$ \\
\hline M0 & 167 & 89 & 78 & 46.7 & & \\
\hline M1 & 53 & 8 & 45 & 84.9 & & \\
\hline TNM staging & & & & & 26.416 & $<0.001$ \\
\hline $\mathrm{I}+\mathrm{II}$ & 71 & 49 & 22 & 31 & & \\
\hline $\mathrm{III}+\mathrm{IV}$ & 149 & 48 & 101 & 67.8 & & \\
\hline YAP1 expression & & & & & 1.8712 & 0.171 \\
\hline Low & 41 & 22 & 19 & 46.3 & & \\
\hline High & 179 & 75 & 104 & 58.1 & & \\
\hline
\end{tabular}

HPR high positive rate, Histol. histological, Papi. papillary, ade. adenocarcinoma, diff. differentiation, Undiff. undifferentiated, Car carcinoma, SRC signet ring cell cancer, $L n$. lymph node

Bold values indicates that statistical significance of $P$ value is less than 0.05
Kaplan-Meier analysis showed that overall survival for patients with YAP1 high-expression was significantly worse than in patients with low YAP1 expression $(P=0.001$, Fig. 2n). Patients with low expression of both Nodal and YAP1 (Nodal ${ }^{-} \mathrm{YAP}^{-}$) demonstrated the best outcome. In contrast, patients with high expression of both proteins $\left(\mathrm{Nodal}^{+} \mathrm{YAP}^{+}\right.$) demonstrated the worst outcome (Fig. 2o; Table 3.)

\section{Discussion}

Nodal is a member of the TGF- $\beta$ superfamily that regulates many important aspects of early development, including germ layer specification and axis patterning. While its expression in many adult tissues is down-regulated, it has been shown to be re-expressed in cancer cells, where it 
Table 3 Univariate and multivariate analyses of different prognostic factors in 161 patients with GC

\begin{tabular}{|c|c|c|c|c|c|}
\hline \multirow[t]{2}{*}{ Variable } & \multicolumn{3}{|c|}{ Univariate analysis $^{\mathrm{a}}$} & \multicolumn{2}{|l|}{ Multivariate analysis $^{\mathrm{b}}$} \\
\hline & $n$ & Mean survival (months) & $P$ value & HR $(95 \% \mathrm{CI})$ & $P$ value \\
\hline Borrmann's types & & & 0.289 & $1.113(0.646-1.985)$ & 0.663 \\
\hline $\mathrm{I}+\mathrm{II}$ & 22 & 29.55 & & & \\
\hline $\mathrm{III}+\mathrm{IV}$ & 139 & 32.62 & & & \\
\hline WHO's histology types & & & 0.638 & $1.096(0.913-1.317)$ & 0.324 \\
\hline Papillary. Ade. & 6 & 32.50 & & & \\
\hline Tubular Ade. & & & & & \\
\hline Well Diff. & 11 & 33.80 & & & \\
\hline Moderately Diff. & 56 & 35.29 & & & \\
\hline Poorly Diff. & 70 & 29.71 & & & \\
\hline Un-differentiated car. & 3 & 35.67 & & & \\
\hline Signet ring cell car. & 5 & 16.60 & & & \\
\hline Mucinous Ade. & 10 & 26.07 & & & \\
\hline Lauren's types & & & 0.354 & $1.055(0.747-1.492)$ & 0.760 \\
\hline Intestinal & 56 & 35.11 & & & \\
\hline Diffuse & 77 & 31.41 & & & \\
\hline Mixed & 28 & 28.13 & & & \\
\hline Depth of invasion & & & 0.001 & $1.886(0.895-3.975)$ & 0.095 \\
\hline $\mathrm{T} 1+\mathrm{T} 2$ & 28 & 43.96 & & & \\
\hline $\mathrm{T} 3+\mathrm{T} 4$ & 133 & 29.54 & & & \\
\hline Ln metastasis & & & 0.001 & $0.450(0.208-0.974)$ & 0.043 \\
\hline No & 43 & 41.06 & & & \\
\hline $\mathrm{N} 1-3$ & 118 & 28.64 & & & \\
\hline TNM staging & & & $<0.001$ & $6.999(2.754-17.786)$ & $<0.001$ \\
\hline $\mathrm{I}+\mathrm{II}$ & 52 & 46.15 & & & \\
\hline $\mathrm{III}+\mathrm{IV}$ & 109 & 25.23 & & & \\
\hline Nodal protein expression & & & $<0.001$ & $2.487(1.546-4.001)$ & $<0.001$ \\
\hline Low & 80 & 40.90 & & & \\
\hline High & 81 & 23.58 & & & \\
\hline YAP1 protein expression & & & 0.001 & $2.506(1.195-5.257)$ & 0.015 \\
\hline Low & 29 & 44.90 & & & \\
\hline High & 132 & 29.22 & & & \\
\hline Nodal and YAP1 expression & & & $<0.001$ & & \\
\hline $\mathrm{Nodal}^{-} \mathrm{YAP}^{-}$ & 18 & 50.30 & & & \\
\hline $\mathrm{Nodal}^{-} \mathrm{YAP}^{+}$ & 59 & 37.35 & & & \\
\hline $\mathrm{Nodal}^{+} \mathrm{YAP}^{-}$ & 14 & 39.38 & & & \\
\hline $\mathrm{Nodal}^{+} \mathrm{YAP} 1^{+}$ & 70 & 21.51 & & & \\
\hline
\end{tabular}

Ade Adenocarcinoma, Diff. Differentiated, Car. Carcinoma, Ln. lymph node

${ }^{\text {a }}$ Log-rank test; ${ }^{\text {b }}$ Cox regression model likely promotes tumorigenesis. This claim is supported by data showing that down-regulation of Nodal significantly reduces tumorigenicity of several cancer cell line models (Lee et al. 2010; Quail et al. 2012; Topczewska et al. 2006). YAP1 is a member of the Hippo signaling pathway, which regulates organ size by coordinating cellular proliferation and apoptosis. Overexpression of YAP1 has been shown to correlate with disease progression and poor prognosis of gastric cancer. Previous studies have reported cross talk between components of the Hippo and TGF- $\beta$ pathways, both at the level of intracellular signaling and transcriptional regulation (Grannas et al. 2015). Despite this, interplay between these two pathways in gastric adenocarcinoma has not been thoroughly examined. To gain insight into the role of Nodal and YAP1 in gastric cancer, we examined their expression in a panel of patient tumor samples and matched normal tissue. We found that elevated expression of either Nodal or YAP1 is associated with 
several clinicopathological variables. Additionally, patients with tumors expressing high levels of both Nodal and YAP1 displayed the worse prognosis. The data we present here suggest that inhibition of the Hippo and TGF- $\beta$ pathways in gastric adenocarcinoma may have some therapeutic benefit.

We first examined mRNA and protein expression of Nodal and YAP1 in a panel of gastric adenocarcinoma patient samples and matched normal tissue. We measured significant overexpression of Nodal and YAP1 at both the mRNA and protein levels in tumors compared to normal tissue. These findings suggest that these factors and their respective signaling pathways might be drivers of disease progression. This is supported by earlier work showing that YAP1 expression correlates with progression, metastasis, and poor prognosis in patients with gastric carcinoma (Hu et al. 2014). Moreover, Nodal has been found to be up-regulated in various cancers, including breast cancer (Kirsammer et al. 2004), endometrial cancer (Cruz et al. 2015), pancreatic cancer (Kong et al. 2015), and melanoma (Strizzi et al. 2015). The melanoma study is particularly interesting since the authors showed no expression of Nodal in normal skin but increased nodal expression in approximately $60 \%$ of invasive melanoma cases. These data correlate with the findings we present here and support the fact that Nodal expression is associated with advanced disease state and overall prognosis.

We expanded on these initial observations by examining the potential correlation between Nodal and YAP1 expression and several clinicopathological variables. Nodal expression correlated with depth of tumor invasion, lymph node metastasis, and distant metastasis. Furthermore, we found that either high Nodal or YAP1 expression served as an independent risk factor for GAC prognosis. Previous work from our group has already established that YAP1 also correlates with these same clinicopathological variables (Hu et al. 2014). Importantly, we discovered that patients with tumors in which both Nodal and YAP1 were expressed at high levels had the worse overall prognosis. These data suggest that these two factors or their respective signaling pathways may cooperate to promote disease progression. Since we did not detect significant correlation between expression of Nodal and YAP1 themselves, it is possible that the Hippo and TGF- $\beta$ pathways interact downstream at the signal transduction level to enhance disease pathogenesis. Additional work is required to better understand the nature of the cross talk between these two pathways.

The work we present here is not without its limitations. Most notably, the data we present here do not address the reason why gastric adenocarcinomas expressing both Nodal and YAP1 at high levels have the worse overall prognosis. Second, sample size is limited, which prevents us from drawing vast conclusions; additional studies with more samples would enhance the work we present here. Third, follow-up information for all patients after surgery was not available; this may have introduced bias into our analysis. Finally, the data we present are largely correlative. Follow-up studies in either cell lines or animal models in which levels of Nodal or YAP1 could be manipulated would help establish a possible causal role for either of these factors in disease progression.

In summary, we show that Nodal and YAP1 are increased in gastric adenocarcinomas compared to normal tissue. Elevated Nodal expression is a marker of poor prognosis in gastric cancer. Patient outcome is further worsened if Nodal and YAP1 are both expressed in the same tumor. The data we present here suggest that inhibition of Nodal signaling may represent a new therapeutic strategy for the treatment of gastric adenocarcinoma.

Acknowledgments This work was supported by the National Natural Science Foundation of China (Nos. 81071650; 30973503), Special foundation for Science and Technology Program in Liaoning Province, China (2013225303-103; 2011404013-3), and the Supporting Project for Climbing Scholars in Liaoning Provincial Universities, China (2009-2012).

Authors' contributions Yan Xin conceived and supervised this study and revised the manuscript. Ping Li and Dan Sun made equal contributions to this article, including performing experiments, interpreting data, and drafting the manuscript. Xiaoting Li and Yingjian were responsible for the statistical analyses. Wenhui Li, Jing Zhao, Ying Wang, and Huan Wang were responsible for collecting tissue specimens and relevant clinicopathological data. All authors read and approved the final manuscript.

\section{Compliance with ethical standards}

Conflict of interest The authors declare that they have no conflict of interest.

Ethical approval This article does not contain any studies with human participants or animals performed by any of the authors.

Informed consent Informed consent was obtained from all individual participants included in the study.

Open Access This article is distributed under the terms of the Creative Commons Attribution 4.0 International License (http://creativecommons.org/licenses/by/4.0/), which permits unrestricted use, distribution, and reproduction in any medium, provided you give appropriate credit to the original author(s) and the source, provide a link to the Creative Commons license, and indicate if changes were made.

\section{References}

Aykul S, Ni W, Mutatu W, Martinez-Hackert E (2015) Human Cerberus prevents nodal-receptor binding, inhibits nodal signaling, and suppresses nodal-mediated phenotypes. PLoSOne 10:e0114954. doi:10.1371/journal.pone.0114954

Cruz CD, Del Puerto HL, Rocha AL, Cavallo IK, Clarizia AD, Petraglia F, Reis FM (2015) Expression of Nodal, Cripto, SMAD3, phosphorylated SMAD3, and SMAD4 in the proliferative 
endometrium of women with endometriosis. Reprod Sci 22:527533. doi:10.1177/1933719114549855

Hu X, Xin Y, Xiao Y, Zhao J (2014) Overexpression of YAP1 is correlated with progression, metastasis and poor prognosis in patients with gastric carcinoma. Pathol Oncol Res 20(4):805-811

James D, Levine AJ, Besser D, Hemmati-Brivanlou A (2005) TGFbeta/activin/nodal signaling is necessary for the maintenance of pluripotency in human embryonic stem cells. Development 132:1273-1282. doi:10.1007/s00421-008-0955-8

Kirsammer G, Strizzi L, Margaryan NV, Gilgur A, Hyser M, Atkinson J, Kirschmann DA, Seftor EA, Hendrix MJ (2004) Nodal signaling promotes a tumorigenic phenotype in human breast cancer. Semin Cancer Biol 29:40-50. doi:10.1016/j. semcancer.2014.07.007

Kong B, Wang W, Esposito I, Friess H, Michalski CW, Kleeff J (2015) Increased expression of Nodal correlates with reduced patient survival in pancreatic cancer. Pancreatology 15:156-161. doi:10.1016/j.pan.2015.02.001

Lee CC, Jan HJ, Lai JH, Ma HI, Hueng DY, Lee YC, Cheng YY, Liu LW, Wei HW, Lee HM (2010) Nodal promotes growth and invasion in human gliomas. Oncogene 29(21):3110-3123. doi:10.1038/onc.2010.55

Postovit LM, Margaryan NV, Seftor EA, Kirschmann DA, Lipavsky A, Wheaton WW, Abbott DE, Seftor RE, Hendrix MJ (2008) Human embryonic stem cell microenvironment suppresses the tumorigenic phenotype of aggressive cancer cells. Proc Natl Acad Sci USA 105:4329-4334. doi:10.1073/pnas.0800467105

Quail DF, Zhang G, Walsh LA, Siegers GM, Dieters-Castator DZ, Findlay SD, Broughton H, Putman DM, Hess DA, Postovit LM (2012) Embryonic morphogen nodal promotes breast cancer growth and progression. PLoS ONE 7(11):e48237. doi:10.1371/ journal.pone. 0048237
Röttinger E, DuBuc TQ, Amiel AR, Martindale MQ (2015) Nodal signaling is required for mesodermal and ventral but not for dorsal fates in the indirect developing hemichordate, Ptychodera flava. Biol Open 4:830-842. doi:10.1242/bio.011809

Soukup V, Yong LW, Lu TM, Huang SW, Kozmik Z, Yu JK (2015) The Nodal signaling pathway controls left-right asymmetric development in amphioxus. Evodevo 6:5. doi:10.1186/2041-9139-6-5

Strizzi L, Sandomenico A, Margaryan NV, Focà A, Sanguigno L, Bodenstine TM, Chandler GS, Reed DW, Gilgur A, Seftor EA, Seftor RE, Khalkhali-Ellis Z, Leonardi A, Ruvo M, Hendrix MJ (2015) Effects of a novel Nodal-targeting monoclonal antibody in melanoma. Oncotarget 6:34071-34086. doi:10.18632/ oncotarget.6049

Sudol M (1994) Yes-associated protein (YAP65) is a proline-rich phosphoprotein that binds to the $\mathrm{SH} 3$ domain of the Yes protooncogene product. Oncogene 9:2145-2152

Grannas K, Arngården L, Lönn P, Mazurkiewicz M, Blokzijl A, Zieba A, Söderberg O (2015) Crosstalk between Hippo and TGF $\beta$ : Subcellular Localization of YAP/TAZ/Smad Complexes. J Mol Biol 427(21):3407-3415

Topczewska JM, Postovit LM, Margaryan NV, Sam A, Hess AR, Wheaton WW, Nickoloff BJ, Topczewski J, Hendrix MJ (2006) Embryonic and tumorigenic pathways converge via Nodal signaling: role in melanoma aggressiveness. Nat Med 12(8):925-932

Zhang H, von Gise A, Liu Q, Hu T, Tian X, He L, Pu W, Huang X, He L, Cai CL, Camargo FD, Pu WT, Zhou B (2014) Yap1 is required for endothelial to mesenchymal transition of the atrioventricular cushion. J Biol Chem 289(27):18681-18692

Vandeputte C, Docherty JR (2002) Vascular actions of 3,4-methylenedioxymethamphetamine in alpha(2A/D)-adrenoceptor knockout mice. Eur J Pharmacol 457(1):45-49 